\title{
A SIMPLE GEOMETRIC PROOF THAT COMONOTONIC RISKS HAVE THE CONVEX-LARGEST SUM
}

\author{
BY

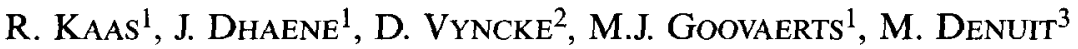

\begin{abstract}
In the recent actuarial literature, several proofs have been given for the fact that if a random vector $\left(X_{1}, X_{2}, \ldots, X_{n}\right)$ with given marginals has a comonotonic joint distribution, the sum $X_{1}+X_{2}+\cdots+X_{n}$ is the largest possible in convex order. In this note we give a lucid proof of this fact, based on a geometric interpretation of the support of the comonotonic distribution.
\end{abstract}

\section{INTRODUCTION}

Up to a few years ago, when dealing with stochastic orderings, actuarial risk theory has focused on single risks or sums of independent risks. Here risks denote non-negative random variables such as they occur in the individual and the collective model, see, e.g., Kaas et al. (1994, 2001). Lately, with an eye on financial actuarial applications, the attention has shifted to sums $X_{1}+X_{2}+\cdots$ $+X_{n}$ of random variables that may also have negative values. Moreover, their independence is no longer required. Only the marginal distributions are assumed to be fixed. A central result is that in this situation, the sum of the components $X_{1}+X_{2}+\cdots+X_{n}$ is the riskiest if the joint distribution of the random variables $X_{i}$ is comonotonic. This means that the support of $\left(X_{1}, X_{2}, \ldots, X_{n}\right)$ has the property that each two points in this support have ordered components.

Perhaps due to the fact that so far no standard actuarial textbook has dealt with this topic, independent proofs of this result have appeared in several papers lately. Since Schmeidler (1986), Yaari (1987) and Roëll (1987), the concept of comonotonicity has played an important role in the economic theories of decision under risk and uncertainty. Hoeffding (1940) and Fréchet (1951) studied the comonotonic distribution without actually proving the sum of the components to be riskier. As far as we know, this result was first mentioned in the actuarial literature in Heilmann (1986), who attributes it to Meilijson

1 University of Amsterdam and K.U. Leuven.

2 K.U. Leuven.

3 U.C.L., Louvain-la-Neuve.

ASTIN BULLETIN, Vol. 32, No. 1, 2002, pp. 71-80 
and Nadas (1979). Proofs of it involving the more general concept of supermodularity are contained in Müller (1997) (see also Bäuerle and Müller, 1998), Goovaerts and Dhaene (1999) and Goovaerts and Redant (1999). In Goovaerts and Kaas (2002) a proof is given involving limits for random variables with finitely many values. Proofs for the special case $n=2$ and for the individual life model (two-point distributions) are to be found in Dhaene \& Goovaerts (1996) and Dhaene \& Goovaerts (1997) respectively. Goovaerts et al. (2000) considers only continuous random variables. In Kaas et al. (2000), a general proof is given using an extension of the notion inverse distribution function. Dhaene et al. (2000) prove a slightly more general result. See also Dhaene and Denuit (1999), Wang and Dhaene (1998) and Wang and Young (1998).

A drawback of all these proofs is that none of them contributes a lot to the intuitive understanding of the concepts involved. In this short note, we give a transparent proof which is based on the geometric properties of the comonotonic support, and which we think is more suitable for classroom use. Also, it might inspire others to work on applications of comonotonic risks.

In Section 2, we define the order concepts used, convex order and stoploss order. In Section 3, we describe the support and the joint cdf of the comonotonic distribution. Section 4 provides simple continuous and discrete examples. Section 5 finally gives the proof that comonotonicity implies a convex-largest sum of the components of a random vector.

\section{CONVEX ORDER AND STOP-LOSS ORDER}

The natural ordering concept in actuarial science is the stop-loss order. A random variable $X$ is less than $Y$ in stop-loss order, written $X \leq_{S L} Y$, if their net stop-loss premiums satisfy

$$
E\left[(X-d)_{+}\right] \leq E\left[(Y-d)_{+}\right] \text {for all real } d .
$$

This order has many useful invariance properties. For instance, stop-loss order survives the operations of convolution and compounding on non-negative random variables (risks), and stop-loss larger claims lead to increased ruin probability and higher zero-utility premiums for risk averse decision makers. Risk $X$ is preferred over $Y$ either because it represents a smaller loss, or because it is less spread. See for instance Kaas et al. $(1994,2001)$ in the framework of actuarial sciences.

The quantity $E\left[(X-d)_{+}\right]$represents the expected loss over $d$. With stability in mind, not only excessively large positive values of a random variable are unattractive, but also negative ones. Hence $E\left[(-X-t)_{+}\right]$should be small too. So in that case, random variable $X$ is preferred over $Y$ if for all real $d=$ $-t$, both

$$
\begin{aligned}
& E\left[(X-d)_{+}\right] \leq E\left[(Y-d)_{+}\right], \text {and } \\
& E\left[(d-X)_{+}\right] \leq E\left[(d-Y)_{+}\right]
\end{aligned}
$$


If this holds, we say that $X$ is less than $Y$ in convex order, written as $X \leq_{c x} Y$. Note that adding $d$ to the first set of inequalities and letting $d \rightarrow-\infty$ leads to $E[X] \leq E[Y]$. Subtracting $d$ in the second set of inequalities and letting $d \rightarrow+\infty$ produces $E[X] \geq E[Y]$, hence $E[X]=E[Y]$ must hold for two random variables to be comparable in convex order. On the other hand, the first set of inequalities together with equal means can be shown to imply the second set.

Stop-loss order can be shown to be the same as having ordered expected values $E[f(X)]$ for all non-decreasing convex functions $f(\cdot)$, see e.g. Goovaerts et al. (1990). Hence it represents the common preferences of all risk-averse decision makers. On the other hand, convex order is the same as ordered expectations for all convex functions, see e.g. the standard work on stochastic orders in a more general framework, Shaked and Shanthikumar (1994). This is of course where the name convex order comes from. In a utility context, it represents the common preferences of all risk-averse decision makers between random variables with equal mean. The proof that convex order implies ordered expectations of convex functions generally relies on the classical argument that any convex function can be obtained as the uniform limit of a sequence of piecewise linear functions, each of them being expressible as a linear combination of functions $(x-t)_{+}$and $(t-x)_{+}$. A somewhat simpler proof, relying on partial integration, is given below, not because it is new, but just to keep the paper self-contained.

Lemma 1. If $X \leq_{c x} Y$ and $f(\cdot)$ is convex, then $E[f(X)] \leq E[f(Y)]$.

Proof. Consider the function $g(x)=f(x)-f(a)-(x-a) f^{\prime}(a)$, with $a$ some point where $f(\cdot)$ is differentiable. Since $E[X]=E[Y]$, the inequality $E[f(X)] \leq E[f(Y)]$ is equivalent to $E[g(X)] \leq E[g(Y)]$. Write $F(x)=\operatorname{Pr}[X \leq x]$ and $F(x)=\mathrm{I}-F(x)$. Since $g(a)=g^{\prime}(a)=0$, the integrated terms below vanish, so by four partial integrations we get

$$
\begin{aligned}
E[g(X)] & =\int_{-\infty}^{a} g(x) d F(x)-\int_{a}^{+\infty} g(x) d \bar{F}(x) \\
& =-\int_{-\infty}^{a} g^{\prime}(x) F(x) d x+\int_{a}^{+\infty} g^{\prime}(x) \bar{F}(x) d x \\
& =\int_{-\infty}^{a} E\left[(x-X)_{+}\right] d g^{\prime}(x)+\int_{a}^{+\infty} E\left[(x-X)_{+}\right] d g^{\prime}(x)
\end{aligned}
$$

from which the result immediately follows, because by the convexity of $f(\cdot)$ and also $g(\cdot)$, we have $d g^{\prime}(x) \geq 0$ for all $x$.

\section{THE SUPPORT OF THE COMONOTONIC DISTRIBUTION}

We start by defining comonotonicity of a set of real $n$-vectors in $R^{n}$. When the support of a random vector is a comonotonic set, also the random vector itself as well as its distribution are called comonotonic.

Definition 1. The set $S$ in $R^{n}$ is said to be comonotonic, if, for all $\left(y_{1}, y_{2}, \ldots, y_{n}\right)$ and $\left(z_{1}, z_{2}, \ldots, z_{n}\right)$ in this set, $y_{i}<z_{i}$ for some $i$ implies $y_{j} \leq z_{j}$ for all $j$. 
Notice that a comonotonic set is a "thin" set. Since the upper left and lower right corners of a rectangle may not both be in it, it must be (a subset of) a curve that is monotonically non-decreasing in each component. It cannot contain any subset of dimension larger than 1 .

Proposition 2. The connected closure $\bar{S}$ of the comonotonic set $S$ is a continuous curve which is comonotonic.

Proof. The set $S$ consists of a series of connected closed curves, possibly containing just one point. Now, connect the endpoints of consecutive curves by straight lines. Note that this has to be done only countably many times. We are left with $\bar{S}$, which is a continuously increasing curve in $R^{n}$.

The set $\bar{S}$, having no more missing pieces, can be parametrized by non-decreasing continuous functions such that

$$
\bar{S}=\left\{\left(f_{1}(z), \ldots, f_{n}(z)\right) \mid-\infty<z<\infty\right\} .
$$

Hence, it may be traversed in an upward direction (with increasing $z$ ).

Proposition 3. Let the support of random vector $\left(Y_{1}, Y_{2}, \ldots, Y_{n}\right)$ be contained in the connected closed curve $\bar{S}$. Then the joint cdf of $\left(Y_{1}, Y_{2}, \ldots, Y_{n}\right)$ must have the following form:

$$
\operatorname{Pr}\left[Y_{1} \leq y_{1}, \ldots, Y_{n} \leq y_{n}\right]=\min _{j=1, \ldots, n} \operatorname{Pr}\left[Y_{j} \leq y_{1}\right] .
$$

Proof. We are looking for the total probability of the region $R=R_{1} \cap R_{2} \cap \cdots$ $\cap R_{n}$, where $R_{j}, j=1,2, \ldots, n$ is defined as the region $\left\{t \in R^{n} \mid t_{j} \leq y_{j}\right\}$. Excluding the trivial cases $\bar{S} \subset R$ and $\bar{S} \cap R=\varnothing$, as vector $s$ traverses $\bar{S}$ in the upward direction, it must reach one of the boundary planes $\left\{t \in R^{n} \mid t_{j}=y_{j}\right\}, j=1,2$, $\ldots, n$ of this region first. Let $k$ be the index corresponding to this boundary plane. Then, $\operatorname{Pr}\left[Y_{k} \leq y_{k}\right]=\min _{j} \operatorname{Pr}\left[Y_{j} \leq y_{j}\right]$ and $\bar{S} \cap R=\bar{S} \cap R_{k}$ are obvious from the geometric properties of $\bar{S}$. Hence the event $Y_{1} \leq y_{1}, \ldots, Y_{n} \leq y_{n}$ has the same probability as $Y_{k} \leq y_{k}$, and the proof is completed.

For instance in Figure 2 below, $\bar{S}$ is the dotted line. To compute the joint cdf at $\left(\frac{1}{2}, \frac{3}{2}\right)$, observe that going along $\bar{S}$ upwards, border $x=\frac{1}{2}$ is crossed at $\left(\frac{1}{2}\right.$, 1 ), while the other border $y=\frac{3}{2}$ is crossed only at $\left(\frac{3}{2}, \frac{3}{2}\right)$. The set of points with positive probability to the left of and below $\left(\frac{1}{2}, \frac{3}{2}\right)$ coincides with the corresponding set to the left of $x=\frac{1}{2}$.

Consider some cumulative univariate distribution function $F$. It is wellknown that if $U \sim$ Uniform $(0,1)$, the random variable $F^{-1}(U)$ is distributed according to $F$ (probability integral transform). Note that it is irrelevant how we define $y=F^{-1}(u)$ for arguments $u$ where there is an ambiguity, i.e., where $F(y)=u$ holds for an interval of $y$-values. For the same reason that the cdf of a random variable can have only countably many jumps, it can be shown that there can only be countably many such horizontal segments. [To see this, observe that, in the interval $\left[-2^{n}, 2^{n}\right]$, there are only finitely many intervals with a length over $2^{-n}$ where $F(y)$ is constant, and let $n \rightarrow \infty$.] Hence, if $g(\cdot)$ 
and $h(\cdot)$ are two different choices for the inverse cdf, $g(U)$ and $h(U)$ will be equal with probability one. The customary choice is taking $F^{-1}(u)$ to be the lefthand endpoint of the interval of $y$-values (generally containing one point only) with $F(y)=u$. Then, $F^{-1}(\cdot)$ is non-decreasing and continuous from the left.

Now consider any random vector $\left(X_{1}, X_{2}, \ldots, X_{n}\right)$. We have:

Proposition 4. The following random vector has a comonotonic support, and moreover, it has the same marginal distributions as $\left(X_{1}, X_{2}, \ldots, X_{n}\right)$ :

$$
\left(Y_{1}, Y_{2}, \ldots, Y_{n}\right)=\left(F_{X_{1}}^{-1}(U), F_{X_{2}}^{-1}(U), \ldots, F_{X_{n}}^{-1}(U)\right) \text {. }
$$

Proof. If $\left(y_{1}, y_{2}, \ldots, y_{n}\right)$ and $\left(z_{1}, z_{2}, \ldots, z_{n}\right)$ are in the support of $\left(Y_{1}, Y_{2}, \ldots, Y_{n}\right)$ with $F_{X_{i}}^{-1}(u)=y_{i}<z_{i}=F_{X_{i}}^{-1}(v)$, then $u<v$ must hold, and hence $\mathrm{y}_{j} \leq z_{j}$ for all $j=1$, $2, \ldots, n$.

For the marginals, we have $\operatorname{Pr}\left[F_{X_{i}}^{-1}(U) \leq x\right]=\operatorname{Pr}\left[U \leq F_{X_{i}}(x)\right]=F_{X_{i}}(x)$ for all $x$.

The support of $\left(Y_{1}, Y_{2}, \ldots, Y_{n}\right)$ as in Proposition 4 is the closure of the set

$$
\left\{\left(F_{X_{1}}^{-1}(u), F_{X_{2}}^{-1}(u), \ldots, F_{X_{n}}^{-1}(u)\right) \mid 0<u<1\right\} .
$$

Note that, by Proposition 3, any other comonotonic random vector with the same marginals as $\left(Y_{1}, Y_{2}, \ldots, Y_{n}\right)$ has the same cdf. In this sense, $\left(F_{X_{1}}^{-1}(U), F_{X_{2}}^{-1}(U), \ldots\right.$, $\left.F_{X_{n}}^{-1}(U)\right)$ is the unique comonotonic random vector with the same marginals as $\left(X_{1}, X_{2}, \ldots, X_{n}\right)$.

The following result can be found in Hoeffding (1940) and Fréchet (1951). See also Bäuerle and Müller (1998). In our setup, it can be deduced directly from Propositions 3 and 4.

Corollary 5. The joint $c d f$ of the comonotonic random vector $\left(Y_{1}, Y_{2}, \ldots, Y_{n}\right)$ with the same marginal distributions as $\left(X_{1}, X_{2}, \ldots, X_{n}\right)$ satisfies:

$$
\operatorname{Pr}\left[Y_{1} \leq y_{1}, \ldots, Y_{n} \leq y_{n}\right]=\min _{j=1, \ldots, n} \operatorname{Pr}\left[X_{j} \leq y_{j}\right] .
$$

Note that this proposition implies that the comonotonic cdf is as large as it can possibly be while still having the required marginal distributions. It is equal to an upper bound for all joint cdfs with these marginal distributions. By the same token, the joint probability of all $Y_{j}$ having large values is also maximized. The probability of having some $Y_{j}$ large, some small, is minimized, thus eliminating hedging possibilities. In this light, it is easy to see why the sum $Y_{1}+Y_{2}+\cdots+Y_{n}$ is as variable as possible when the $Y_{j}$ are comonotonic, see Section 5.

\section{TWO SIMPLE EXAMPLES}

First, we give a continuous example with $n=3$. Let $X \sim$ Uniform on the set $\left(0, \frac{1}{2}\right) \cup\left(1, \frac{3}{2}\right), Y \sim \operatorname{Beta}(2,2)$ and $Z \sim \operatorname{Normal}(0,1)$. The support of the comonotonic distribution is the set 


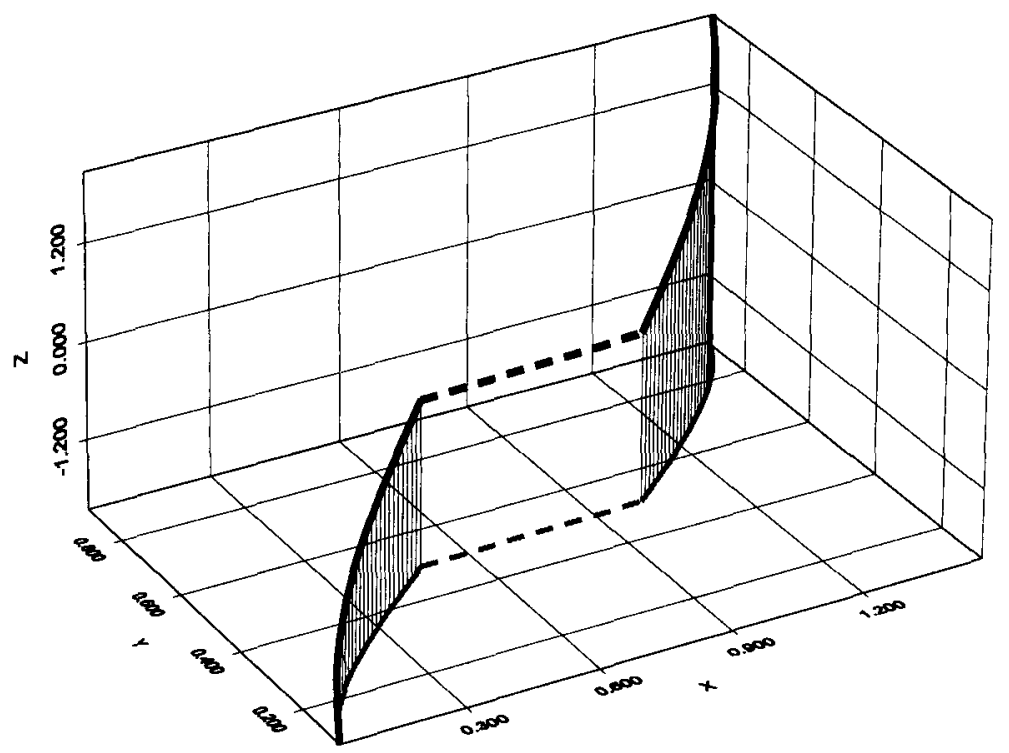

Figure 3: A continuous example with $n=3$.

$$
\left\{\left(F_{X}^{-1}(u), F_{Y}^{-1}(u), F_{Z}^{-1}(u)\right) \mid 0<u<1\right\} .
$$

See Figure 1. Actually, not all of the support is depicted. The part left out corresponds to $u \notin(\Phi(-2), \Phi(2))$ and extends along the asymptotes, the vertical lines $(0,0, z)$ and $\left(\frac{3}{2}, 1, z\right)$. The thick continuous line is $S$, while the dotted line is the straight line needed to make $S$ into the connected curve $\bar{S}$. Note that $F_{X}$ has a horizontal segment between $\frac{1}{2}$ and 1 . The projection of $\bar{S}$ along the $z$-axis can also be seen to constitute an increasing curve, as do projections along the other axes.

For a discrete example, take $X \sim \operatorname{Uniform}\{0,1,2,3\}$ and $Y \sim \operatorname{Binomial}\left(3, \frac{1}{2}\right)$. It is easy to verify that

$$
\begin{aligned}
\left(F_{X}^{-1}(u), F_{Y}^{-1}(u)\right) & =(0,0) \text { for } 0<u<\frac{1}{8}, \\
& =(0,1) \text { for } \frac{1}{8}<u<\frac{2}{8}, \\
& =(1,1) \text { for } \frac{2}{8}<u<\frac{4}{8}, \\
& =(2,2) \text { for } \frac{4}{8}<u<\frac{6}{8}, \\
& =(3,2) \text { for } \frac{6}{8}<u<\frac{7}{8}, \\
& =(3,3) \text { for } \frac{7}{8}<u<1 .
\end{aligned}
$$

The support $S$ of the comonotonic distribution is just these six points, and the curve $\bar{S}$ arises by simply connecting them consecutively with straight lines, the dotted lines in Figure 2. At the boundaries of the intervals for $u$, one may 


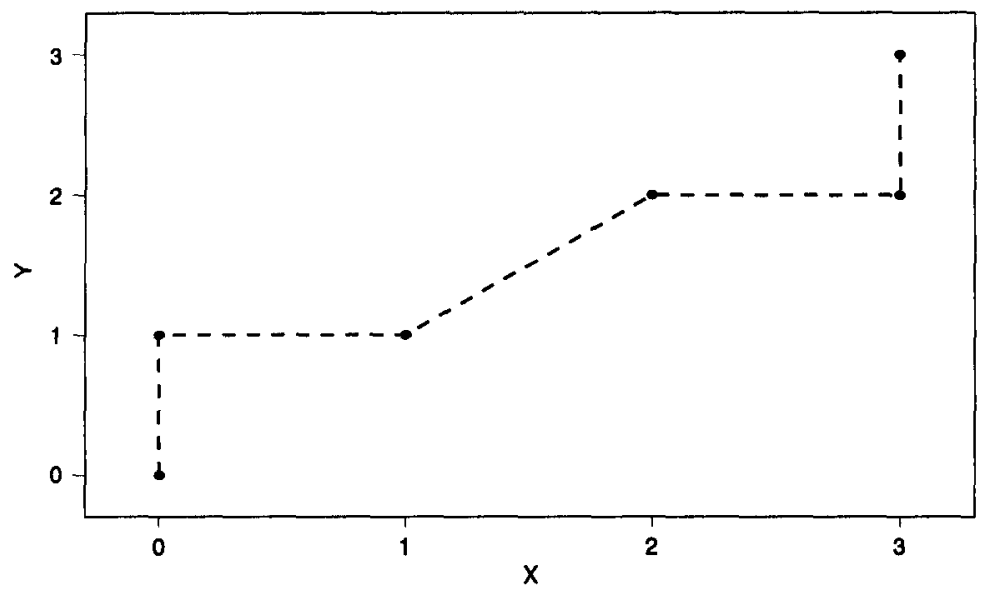

FIGURE 2: A discrete example.

take the limit from either the left or the right. The straight line connecting $(1,1)$ and $(2,2)$ is not along one of the axes. This happens because at level $u=\frac{1}{2}$, both $F_{X}(y)$ and $F_{Y}(y)$ have horizontal segments. Note that any non-decreasing curve connecting $(1,1)$ and $(2,2)$ would have led to a feasible $\hat{S}$. These last two points have probability $\frac{1}{4}$, the other points $\frac{1}{8}$.

\section{COMONOTONICITY AND CONVEX ORDERED SUMS}

After all this work, harvesting our main result is quite easy.

Theorem 6. If the random vector $\left(Y_{1}, Y_{2}, \ldots, Y_{n}\right)$ is comonotonic and has the same marginals as $\left(X_{1}, X_{2}, \ldots, X_{n}\right)$, then

$$
X_{1}+X_{2}+\cdots+X_{n} \leq_{c x} Y_{1}+Y_{2}+\cdots+Y_{n}
$$

Proof. It suffices to prove $\leq_{S L}$, since it is obvious that the means of these two random variables are equal. The following holds for all $\left(x_{1}, x_{2}, \ldots, x_{n}\right)$ when $d_{1}+d_{2}+\cdots+d_{n}=d$ :

$$
\begin{aligned}
& \left(x_{1}+x_{2}+\cdots+x_{n}-d\right)_{+} \\
= & \left\{\left(x_{1}-d_{1}\right)+\left(x_{2}-d_{2}\right)+\cdots+\left(x_{n}-d_{n}\right)\right\}_{+} \\
\leq & \left\{\left(x_{1}-d_{1}\right)_{+}+\left(x_{2}-d_{2}\right)_{+}+\cdots+\left(x_{n}-d_{n}\right)_{+}\right\}_{+} \\
= & \left.\left(x_{1}-d_{1}\right)_{+}+\left(x_{2}-d_{2}\right)_{+}+\cdots+\left(x_{n}-d_{n}\right)_{+}\right\}_{+} .
\end{aligned}
$$

Assume that $d$ is such that $0<\operatorname{Pr}\left[Y_{1}+Y_{2}+\ldots+Y_{n} \leq d\right]<1$ holds; if not, the stop-loss premiums of $Y_{1}+Y_{2}+\cdots+Y_{n}$ and $X_{1}+X_{2}+\cdots+X_{n}$ can be seen to be equal. The connected curve $\bar{S}$ containing the support $S$ of the comonotonic 
random vector $\left(Y_{1}, Y_{2}, \ldots, Y_{n}\right)$ points upwards in all coordinates, so it is obvious that $\bar{S}$ has exactly one point of intersection with the hyperplane $\left\{\left(x_{1}, \ldots\right.\right.$, $\left.\left.x_{n}\right) \mid x_{1}+\ldots+x_{n}=d\right\}$. Let's assume from now on that $\left(d_{1}, d_{2}, \ldots, d_{n}\right)$ is this point of intersection. But then for all points $\left(y_{1}, y_{2}, \ldots, y_{n}\right)$ in the support $S$ of $\left(Y_{1}, Y_{2}, \ldots, Y_{n}\right)$, we have the following equality:

$$
\left(y_{1}+y_{2}+\cdots+y_{n}-d\right)_{+} \equiv\left(y_{1}-d_{1}\right)_{+}+\left(y_{2}-d_{2}\right)_{+}+\cdots+\left(y_{n}-d_{n}\right)_{+} .
$$

This is because for this particular choice of $\left(d_{1}, d_{2}, \ldots, d_{n}\right)$, by the comonotonicity we have that whenever $y_{j}>d_{j}$ for any $j$, we also have $y_{k} \geq d_{k}$ for all $k$; when all $y_{j} \leq d_{j}$, obviously the left hand side is 0 as well.

Now replacing constants by the corresponding random variables in the two relations above and taking expectations, we get, since $X_{j}$ and $Y_{j}$ have the same marginal distribution for all $j$,

$$
\begin{aligned}
& E\left[\left(Y_{1}+Y_{2}+\cdots+Y_{n}-d\right)_{+}\right] \\
= & E\left[\left(Y_{1}-d_{1}\right)_{+}\right]+E\left[\left(Y_{2}-d_{2}\right)_{+}\right]+\cdots+E\left[\left(Y_{n}-d_{n}\right)_{+}\right] \\
= & E\left[\left(X_{1}-d_{1}\right)_{+}\right]+E\left[\left(X_{2}-d_{2}\right)_{+}\right]+\cdots+E\left[\left(X_{n}-d_{n}\right)_{+}\right] \\
\geq & E\left[\left(X_{1}+X_{2}+\cdots+X_{n}-d\right)_{+}\right] .
\end{aligned}
$$

Note that having fixed $\bar{S}$ in a particular instance, in principle we can determine $d_{1}, d_{2}, \ldots, d_{n}$ as in the proof of Theorem 4 for every $d$, and using these we can express the stop-loss premiums of the comonotonic sum in the marginal stop-loss premiums $E\left[\left(X_{i}-d_{i}\right)_{+}\right]$, see the last equality in the proof just above. Also, we have proven the following theorem:

Theorem 7. If the random vector $\left(Y_{1}, Y_{2}, \ldots, Y_{n}\right)$ is comonotonic, then the u-quantiles of the sum of its components are equal to the sum of the u-quantiles of its components:

$$
F_{Y_{1}+\cdots+Y_{n}}^{-1}(u)=F_{Y_{1}}^{-1}(u)+\cdots+F_{Y_{n}}^{-1}(u) .
$$

Proof. For any $d$, choose $\left(d_{1}, d_{2}, \ldots, d_{n}\right)$ as above. If the event $Y_{1}+Y_{2}+\ldots+Y_{n}$ $\leq d$ has probability $u$, the same holds for $Y_{i} \leq d_{i}$ for all $i$. From this, the result immediately follows.

Hence, comonotonic random variables are quantile-additive. Since quantiles are Values-at-Risk, quantile functions may be added to get the VaR for the total portfolio when the subportfolios are comonotonic. If they are not, this procedure merely provides the VaR with a sum that constitutes a convexorder upper bound for the total on all portfolios. Note that this upper bound has the same mean, which means that the corresponding cdf's, as well as their inverses, must cross. Therefore there exist $u$-values for which the actual VaR is larger, but also for which it is lower, so there is no guarantee that the sum of the VaRs is always larger than the VaR for the sum. But since the upper bound has a larger variance, it will tend to have thicker tails, and hence for $u$ close to 1, its VaR will generally be larger. 


\section{ACKNOWLEDGMENTS}

Marc Goovaerts, Jan Dhaene and David Vyncke acknowledge the financial support of the Onderzoeksfonds K.U. Leuven (grant OT/97/6) and of FWO (grant "Actuarial ordering of dependent risks"). We thank the referees for their comments, and David Promislov, Jenny Young and Björn Sundt for pointing out some minor errors and possible enhancements at the Insurance: Mathematics and Economics conference held at Penn State, 2001.

\section{REFERENCES}

BäUerle, N. and MÜLleR, A. (1998) "Modeling and comparing dependencies in multivariate risk portfolios", ASTIN Bulletin 28, 59-76.

DHAENE, J. and DENUIT, M. (1999) "The safest dependency structure among risks", Insurance: Mathematics \& Economics 25, 11-21.

DHAENE, J. and GoovaERTS, M. (1996) "Dependency of risks and stop-loss order", ASTIN Bulletin 26, 201-212.

DhaEne, J. and GoovaerTs, M.J. (1997) "On the dependency of risks in the individual life model", Insurance: Mathematics \& Economics 19, 243-253.

DhaEne, J., WANG, S., Young, V. and GoovaerTs, M.J. (2000) "Comonotonicity and maximal stop-loss premiums", Bulletin of the Swiss Association of Actuaries, 2000(2), 99-113.

Fréchet, M. (1951) "Sur les tableaux de corrélation dont les marges sont données", Ann. Univ. Lyon Sect. A, Series 3, 14, 53-77.

GoovaERTS, M.J. and DHAENE, J. (1999) "Supermodular ordering and stochastic annuities", Insurance: Mathematics \& Economics, 24(3), 281-290.

Goovaerts, M.J., Dhaene, J. and De SChePPER, A. (2000) "Stochastic Upper Bounds for Present Value Functions", Journal of Risk and Insurance Theory, 67.1, 1-14.

GoOvaERTS, M.J. and KAAS, R. (2002) "Some problems in actuarial finance involving sums of dependent risks", Statistica Neerlandica, to appear.

Goovaerts, M.J., KaAs, R., Van Heerwaarden, A.E. and Bauwelinckx, T. (1990) "Effective Actuarial Methods". North-Holland, Amsterdam.

GOOVAERTS, M.J. and REDANT, R. (1999) "On the distribution of IBNR reserves", Insurance: Mathematics \& Economics 25, 1-9.

HoEFFDING, W. (1940) "Masstabinvariante Korrelationstheorie", Schriften des mathematischen Instituts und des Instituts für angewandte Mathematik der Universität Berlin 5, 179-233.

KAAS, R., VAN HEERWAARDEN, A.E. and GoOVAERTS, M.J. (1994) "Ordering of actuarial risks", Institute for Actuarial Science and Econometrics, University of Amsterdam, Amsterdam.

KAAS, R., DhaENE, J. and GoovaerTs, M.J. (2000). "Upper and lower bounds for sums of random variables", Insurance: Mathematics \& Economics 23, 151-168.

KaAs, R., GoovaerTs, M.J., Dhaene, J. and DenutT, M. (2001). "Modern Actuarial Risk Theory", Kluwer, Dordrecht.

MeILusSon, I. and NADAs, A. (1979) "Convex majorization with an application to the length of critical paths", Journal of Applied Probability, 16, 671-677.

MÜlLER, A. (1997) "Stop-loss order for portfolios of dependent risks", Insurance: Mathematics \& Economics 21, 219-223.

RoËLL, A. (1987) "Risk aversion in Quiggin and Yaari's rank-order model of choice under uncertainty", The Economic Journal 97 (Conference 1987), 143-159.

SCHMEIDLER, D. (1986) "Integral representation without additivity", Proceedings of the American Mathematical Society 97, 255-261.

Shaked, M. and Shanthikumar, J.G. (1994) Stochastic orders and their applications. Probability and Mathematical Statistics, Academic Press.

WANG, S. and DHAENE, J. (1998) "Comonotonicity, correlation order and stop-loss premiums", Insurance: Mathematics \& Economics 22, 235-243. 
WANG, S. and YounG, V. (1998) "Ordering risks: expected utility versus Yaari's dual theory of choice under risk". Insurance: Mathematics \& Economics 22, 145-162.

YAARI, M.E. (1987) "The dual theory of choice under risk", Econometrica 55, 95-115.

\section{ROB KAAS}

Faculty of Economics and Econometrics, Dept KE

University of Amsterdam

Roetersstraat 11

1018 WB Amsterdam, The Netherlands

e-mail: robkaas@fee.uva.nl

JAN DHAENE

Center for Risk and Insurance Studies

Catholic University of Leuven

Naamsestraat 69

3000 Leuven, Belgium

e-mail: Jan.Dhaene@econ.kuleuven.ac.be

DAVID VYNCKE

Center for Risk and Insurance Studies

Catholic University of Leuven

Naamsestraat 69

3000 Leuven, Belgium

e-mail: David.Vyncke@econ.kuleuven.ac.be

MARC J. GoOVAERTS

Center for Risk and Insurance Studies

Catholic University of Leuven

Naamsestraat 69

3000 Leuven, Belgium

e-mail: Marc.Goovaerts@econ.kuleuven.ac.be

Michel Deñuit

Institut de Statistique

Université Catholique de Louvain

Voie du Roman Pays 20

1348 Louvain-la-Neuve, Belgium

e-mail:denuit@stat.ucl.ac.be 